\title{
Training on the Job: Behavioral Analysis of Job Interviews in Hospitality
}

\author{
Skanda Muralidhar \\ Idiap Research Institute \\ EPFL, Switzerland \\ smuralidhar@idiap.ch
}

\author{
Jean-Marc Odobez \\ Idiap Research Institute \\ EPFL, Switzerland \\ odobez@idiap.ch
}

\author{
Laurent Son Nguyen \\ Idiap Research Institute \\ EPFL, Switzerland \\ Inguyen@idiap.ch
}

\author{
Marianne Schmid Mast \\ University of Lausanne \\ Switzerland
}

\author{
Denise Frauendorfer \\ University of Lausanne \\ Switzerland \\ denise.frauendorfer@unil.ch
}

Marianne.SchmidMast@unil.ch gatica@idiap.ch

\begin{abstract}
First impressions play a critical role in the hospitality industry and have been shown to be closely linked to the behavior of the person being judged. In this work, we implemented a behavioral training framework for hospitality students with the goal of improving the impressions that other people make about them. We outline the challenges associated with designing such a framework and embedding it in the everyday practice of a real hospitality school. We collected a dataset of 169 laboratory sessions where two role-plays were conducted, job interviews and reception desk scenarios, for a total of 338 interactions. For job interviews, we evaluated the relationship between automatically extracted nonverbal cues and various perceived social variables in a correlation analysis. Furthermore, our system automatically predicted first impressions from job interviews in a regression task, and was able to explain up to $32 \%$ of the variance, thus extending the results in existing literature, and showing gender differences, corroborating previous findings in psychology. This work constitutes a step towards applying social sensing technologies to the real world by designing and implementing a living lab for students of an international hospitality management school.
\end{abstract}

\section{CCS Concepts}

$\bullet$ Applied computing $\rightarrow$ Psychology; Education; •Human-centered computing $\rightarrow$ Empirical studies in $\mathrm{HCI}$;

\section{Keywords}

Social computing; job interview; nonverbal behavior; first impressions; multimodal interaction

Permission to make digital or hard copies of all or part of this work for personal or classroom use is granted without fee provided that copies are not made or distributed for profit or commercial advantage and that copies bear this notice and the full citation on the first page. Copyrights for components of this work owned by others than the author(s) must be honored. Abstracting with credit is permitted. To copy otherwise, or republish, to post on servers or to redistribute to lists, requires prior specific permission and/or a fee. Request permissions from permissions@ acm.org.

ICMI'16, November 12 - 16, 2016, Tokyo, Japan

(C) 2016 Copyright held by the owner/author(s). Publication rights licensed to ACM. ISBN 978-1-4503-4556-9/16/11 . \$15.00

DOI: http://dx.doi.org/10.1145/2993148.2993191

\section{INTRODUCTION}

First impressions are key in the context of organizations, such as during employment interviews, but also while working on jobs requiring strong communication skills such as sales, marketing, or hospitality. First impressions can be defined as the mental image one forms about something or someone after a first encounter. In professional spheres, these initial judgments can influence critical outcomes, such as being hired or promoted. Psychology researchers have shown that people can make accurate inferences about others, even if the amount of information is very limited [3]. Nonverbal behavior has been established as a major channel through which information is communicated; it constitutes a strong basis on which first impressions are formed [20]. Organizational psychologists have studied job interviews for decades, aiming at understanding the relationships among personality, behavior (both verbal and nonverbal), interview ratings/outcomes, and job performance $[11,13,30]$. Until recently, these studies have been conducted based on manual annotations of behavior, which is laborintensive and makes it difficult to scale.

In the last decade, the advent of inexpensive sensors combined with improved perceptual techniques have enabled the possibility to automatically analyze human face-to-face interactions [14, 28]. In the context of job interviews, recent studies have established the feasibility of automatically inferring interview ratings [24] and other related constructs (e.g. engagement, friendliness, or excitement) [23] up to a certain level. Other researchers [17] have extended these works by developing a social coaching system to enable potential job-seekers to train their behavior in order to convey a more positive first impression to recruiters. To this end, they provided feedback to college undergraduate students about their automatically sensed nonverbal behavior, including head gestures, smiles, and prosody. The subjects that obtained feedback via the coaching system showed improved performance during interviews. To our knowledge, little is known on how to implement a training procedure that can be used by individuals and organizations that can systematically benefit from it, such as schools and employment agencies.

The objective of this study is to design and implement a behavioral training procedure for hospitality students in order to improve the first impressions they make on others, including potential clients and recruiters. To this end, we collaborated with an international hospitality management school, and designed a framework to offer their students the possibility to train their social skills in two 
scenarios that are relevant for their future careers, namely job interviews and reception desk situations. Experiments were conducted by embedding them in the hospitality school's regular practice.

The contributions of this work are the following. First, we describe the challenges associated with designing a training framework for students in an international hospitality school. Second, we collected a dataset of 169 simulated job interviews and reception desk interactions (338 interactions in total), which to our knowledge constitutes one of the largest academic datasets of work-related dyadic interactions. Third, we analyzed the relationship between automatically extracted nonverbal cues and various perceived social variables in a correlation analysis and a prediction task, and extended the results found in [24]. We found gender differences that confirmed previous findings in psychology [21]. Fourth, we observed that students overall improved their interview performance during the second laboratory session, suggesting that the feedback they obtained at least partly helped them improve the first impressions that raters formed about them. We see our work as a step towards the use of social sensing in a living lab for the benefit of students of an international hospitality management school.

\section{RELATED WORK}

Researchers in organizational psychology have studied job interviews for decades, uncovering statistical relationships between nonverbal behavior, personality, hireability, and job performance. Regardless of the setting, the impact of nonverbal behavior on perceiver's impressions and judgments has been established [31]. In the context of job interviews, the applicant's nonverbal behavior was shown to influence the hiring decision of the recruiter. For example, Imada and Hankel showed that high amount of eye contact, smiling and other nonverbal behavior had a significant effect on the outcome of the interview [19]. Furthermore, successful applicants were found to make more direct eye contact, produce more facial expressions, smile and nod more than applicants who were rejected [13]. McGovern and Tinsley reported that applicants with loud and modulating voice, extended eye contact, fluent speech, and expressive face were more likely to be hired than the applicants who did not show such behavior [22]. Along the same lines, powerless speech (i.e., speech punctuated with hesitation and lacking conviction) had a negative effect on the impression ratings compared to applicants with speech disorders like stuttering or lisping [12]. Until recently, research in social psychology relied on manual annotations of nonverbal behavior, which is labor-intensive and difficult to scale with respect to either large number of users or different scenarios.

The advent of inexpensive and unobtrusive sensors combined with improved perceptual techniques have enabled the automatic analysis of human interactions. This domain is multidisciplinary and involves speech processing, computer vision, machine learning, and ubiquitous computing. Early works investigated the use of automatically extracted nonverbal cues to predict social constructs as diverse as dominance, leadership, or personality traits in small group interactions [15]. In a context similar to job interviews, Curhan et al. [8] investigated the relationship between audio cues and social outcomes in dyadic job negotiations. Batrinca et al. [5] used a computational approach to predict Big-Five personality traits in self-presentations where participants had to introduce themselves in front of a computer, in a manner similar to job interviews, but without the presence of an interviewer. Nguyen et al. [24] addressed the problem of automatically analyzing employment interviews. This work used automatically extracted nonverbal cues (speaking turns, prosody, head nods, visual activity) to infer five types of hireability variables in a dataset of 62 real job inter- views. Further research also examined the relationships between body activity, personality and hireability using a mixture of automatically and manually extracted features [26]. Naim et al. [23] extended these works by analyzing a dataset of 138 simulated job interviews from 69 internship seeking students, where they extracted cues related to facial expressions, verbal content, and prosody to predict several social variables (e.g., hiring recommendation, engagement, friendliness) and perceived behaviors (e.g., smile, eye contact, speaking rate).

Existing literature in psychology indicates that people can improve their chances of getting hired in a job interview by practicing both their verbal and nonverbal communication [16]. The recent advances in wearable devices with smart interfaces have enabled a new range of possibilities for behavioral training [27]. In the context of public speaking, Google Glass has been used as a headmounted display system to provide real-time feedback on a presenter's posture, openness, body energy, and speech rate sensed using Kinect and external microphone data [9]. In addition to displaying information, Google Glass was also used as an audio sensor to provide automatic real-time feedback on a speaker's speaking rate and energy [33]; however, the data was processed on an external server. These systems have been evaluated on relatively small cohorts $(N \in[15,30])$ consisting of computer science students, and to this day several important questions remain unanswered, such as the implementation of such systems in realistic settings, or the social acceptability of Google Glass. In the context of job interviews, MACH [17] was developed to train social skills and consists of a virtual agent able to read behavioral cues (facial expressions, speech, and prosody) produced by a participant. Additionally, the system provides summary feedback on various nonverbal cues (smiles, head nods, pauses, etc.), as well as what authors called focused feedback, which consists of visualization of certain behaviors over time along the recorded video. The system was tested in a job interview scenario on a cohort of 60 students (plus 30 as control group). While results showed that the group who was given feedback improved their overall interview performance, little is known on how to implement a training procedure that can be used by individuals and organizations, such as schools or employment agencies to systematically benefit from it.

\section{DATA COLLECTION}

\subsection{Context and Challenges}

One of the main objectives of this work was to design and implement a behavioral training framework for students in an international hospitality school offering bachelor and master degrees for English and French-speaking students. The envisioned framework involves students practicing some of their regular work tasks in realistic conditions. We faced many challenges associated with the real-world implementation of a behavioral training program. The hospitality management programs are immersive with students taking classes and conducting practical work in a real hotel, where they rotate among the different services (kitchen, reception, bar, etc.). First, classes had weekly-rotating shifts of practical work and courses. For practical weeks, students did not know their schedules more than two days in advance. Classes did not start semesters at the same period of the year. These factors inherent to the school made the planning of the data collection complex, both at the semester and week levels, requiring a high level of flexibility from our side. Second, as in any bachelor or master level program, students were busy with their usual curricular activities (course assignments, projects, mid-terms, and finals) that took a significant amount of their time. Third, we also faced some challenges related to the relatively young 
age of the students: some of them were not $100 \%$ reliable and did not show up at scheduled sessions, did not reply to emails/SMS, or dropped out during the course of the study. Additionally, although the benefits of participating in the training program was clear to the majority of students, some felt the investment in time was too high to participate.

To address these challenges, we built a living lab located in the same building as the hospitality management school. We had three evenings per week where the lab was open and students could register up to 24 hours in advance for a training session, with a maximal capacity of 12 student-sessions per week. To avoid overloading the student's schedule, we made efforts to make the training program as time-efficient as possible; in total, the complete procedure for each student took 4 hours distributed over 4 weeks on average. Additionally, we targeted students who were starting their semester, because it corresponded to the time where their schoolrelated workload was the lowest.

Multiple modalities were used to advertise the program. Subscription sheets summarizing the study were placed at the student help-desk; 10-minute class presentations were given to each class within the first two weeks following the start of their semesters, where we listed the benefits for them to participate in the training program; e-mails by the academic directors of the school were sent to students; last, some professors advertised the study during their class. Furthermore, we incentivised the students to participate by offering the equivalent of USD 50 upon the completion of the program. Participation in the program was voluntary.

\subsection{Overall Design}

The behavioral training program was designed to be beneficial for the students. To this end, we identified two important situations in the context of hospitality where behavior plays an important role: employment interviews and interactions with customers at a reception desk. The choice of job interview was motivated by its ubiquity in the process of selecting new personnel; furthermore, we believed that a behavioral training on interviews could be beneficial for students in the relatively short-term to get hired for an internship (which students need to complete as part of their degree requirements) or to land their first job. The choice of the interaction with a client at a reception desk was motivated by the fact that it constitutes a standard type of interaction in hospitality; furthermore, beyond the reception desk, hospitality professionals have to be able to communicate with (possibly angry) customers, regardless of the setting. In addition to the two role-plays, an interactive feedback session was included in the training process. This feedback session was held in groups of 3 to 8 students, in which they were given a presentation on first impressions and behavior, watched and discussed video snippets of their recorded interview and desk role-plays, and received written personalized feedback by professionals in human resources and hospitality. Specifically, the procedure included two laboratory sessions, with a feedback session held in-between. Each laboratory session included two roleplays: one job interview and one front desk interaction. Figure 1 displays an overview of the behavioral training framework.

\subsection{Lab Sessions}

Each laboratory session consisted of a job interview and a reception desk role play. The scenarios of lab sessions 1 and 2 were identical to the exception of the reception desk scenario which was slightly modified.

Both the scenarios were recorded using two Kinect v2 devices, one for each protagonist in the interaction. These sensors record standard RGB color and depth data at 30 frames per second, with a

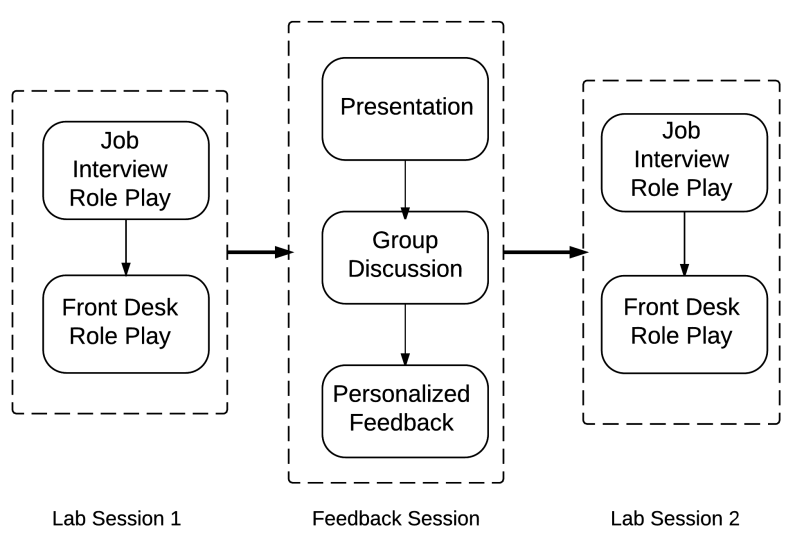

Figure 1: Illustration of the behavioral training procedure.

Job interview questions:

1. Short self-presentation.

2. Motivation for working in the service industry.

3. Past experience requiring attention to details.

4. Past experience where stress was correctly managed.

5. Past experience where adaptability was required.

6. Past experience of calm and tact under stress.

7. Strong/weak points about self.

Figure 2: Interview structure.

spatial resolution of $1920 \times 1080$ for RGB and $512 \times 424$ for depth. Additionally, a Kinect v1 device was placed on top of the reception desk to provide a bird's view of the interaction. For audio, we used a Microcone [1] device which is an array of microphones that automatically performs speaker segmentation based on sound source localization, in addition to recording audio at $48 \mathrm{kHz}$.

Cross-sensor synchronization was obtained by manually adjusting the delay between video data with respect to audio data.

\subsubsection{Job Interview}

The first role-play consists of a job interview, where the student is applying for an internship in a high-end hotel. We used a structured design, i.e., each interview followed the same sequence of questions so that comparisons across subjects could be made. Psychology literature suggests that structured interviews are amongst the most valid tools for selecting applicants [18]. Figure 2 displays the list of questions asked in our study. Students were given the interview questions and five minutes to prepare for the interview, but were not allowed to use notes during the interaction. The interviews were conducted by our research assistants, a pool of seven master's students in psychology and business school. Figure 3 displays a snapshot of the sensor setting used for the job interviews.

\subsubsection{Reception Desk}

The second role-play consisted of an interaction between a receptionist (played by the hospitality student) and a client (played by one of our research assistants) at the reception desk of a highend hotel. Before starting, we instructed the participant about the situation of the role-play: she or he is an intern that started a month ago, it is late and the manager is unavailable, therefore the participant is alone to handle the situation; at one point the bill is handed to the client, and the bill cannot be changed. The scenario was designed in such a way that it starts with a normal phase, where the interaction between the two protagonists is friendly. Once the client gets the bill, the client attitude changes and starts complaining about some issues (e.g. city tax, pay TV, non-free WIFI). Here 


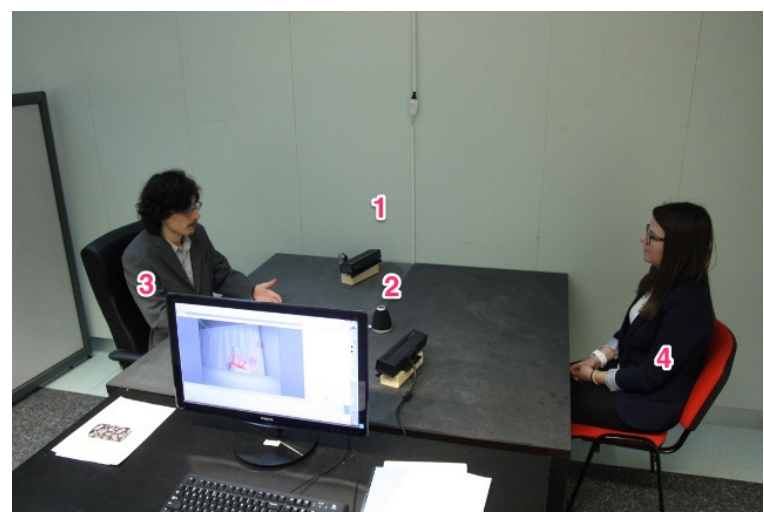

Figure 3: The sensor set-up for job interview setting including the interviewer (3), the participant (4), Microcone (2) and two Kinect devices (1).

the goal of the student is to handle this uneasy situation in the best possible way. The role of the client was played by the same pool of research assistants as the ones who conducted the interviews.

We believe that the reception desk scenario is an interesting addition to the job interviews, as it constitutes a situation where the students perform in a work-like environment. In other words, this scenario could provide an assessment of how well the students perform in a job situation, which could enable us to study the relationship between job interviews and the performance at work. In this study we focused our analysis on the job interviews. The analysis of the desk interactions will be conducted in a separate future study.

\subsection{Feedback Session}

After the first lab session, students participated in a feedback session in groups of three to eight people. First, participants were given a 20-minute presentation on nonverbal behavior and its effect on the first impressions made on others. The presentation was prepared by a researcher in organizational psychology and was given by one of our research assistants.

Then, video snippets of the interactions recorded during the first lab session were watched and discussed by the group of students. Participants were instructed to give constructive comments about several strong points and aspects that could be improved. At least one research assistant was present to moderate the discussion, which was never necessary in practice.

Last, we gave each participant personalized written feedback, which was provided by professionals in human resources (for the job interviews) and hospitality (for the reception desk). The experts were instructed to give constructive feedback on how the students could improve their performance either at the desk or during the interview, based on the full audio-video recording of the interactions during the first lab session.

\subsection{Participants}

In total, we collected 169 job interviews and reception desk interactions, for a total of 338 interactions. In aggregate, the corpus comprises 3040 minutes of recordings, with 1690 minutes of interviews (average interview duration $\approx 10$ minutes) and 1350 minutes for desk (average desk duration $\approx 8$ minutes). To our knowledge, this constitutes one of the largest academic dataset of dyadic interactions collected in an organizational context. 100 students participated in the first lab session, 69 participants completed both lab sessions. The 31 students who did not complete the full training
Table 1: Intra class correlation and descriptive statistics for perceived social variables in job interviews.

\begin{tabular}{|l|l|lll|}
\hline Social Variable & ICC(2,k) & mean & std & skew \\
\hline Professional Skills & & & & \\
Motivated (motiv) & 0.52 & 5.89 & 0.60 & -1.03 \\
Competent (compe) & 0.56 & 6.01 & 0.54 & -1.00 \\
Hardworking (hardw) & 0.54 & 6.06 & 0.55 & -1.07 \\
\hline Social Skills & & & & \\
Sociable (socia) & 0.57 & 5.67 & 0.65 & -0.39 \\
Enthusiastic (enthu) & 0.68 & 5.52 & 0.87 & -0.64 \\
Positive (posit) & 0.60 & 5.70 & 0.72 & -0.46 \\
\hline Communication Skills & & & & \\
Communicative (commu) & 0.60 & 5.82 & 0.71 & -0.79 \\
Concise (consi) & 0.55 & 5.84 & 0.65 & -0.72 \\
Persuasive (persu) & 0.69 & 5.57 & 0.87 & -0.76 \\
\hline Overall Impression & \multirow{4}{|c}{} & & \\
OvImpress & 0.73 & 5.58 & 0.94 & -0.76 \\
\hline
\end{tabular}

procedure either decided to leave the study before or after the feedback session, or did not reply to emails or SMS. Out of the 100 students who participated in at least one lab session, there were 57 females, and the mean age was 20.6 years $(S D=2.47)$. Additionally, because the hospitality management school has programs in both French and English, the two languages are present in the corpus. Out of the 169 sessions, 130 were conducted in French.

\subsection{Annotations}

As the objective of this study was to implement a behavioral training procedure for hospitality student, we were interested in the effects of nonverbal behavior in the perception of various social variables such as first impressions, professional, communication, and social skills.

To observe the initial effect, we showed the first two minutes of the interview video to five raters, who were master's student in psychology. The raters watched the first two minutes of the videos and rated a number of social variables on Likert scale from 1 to 7. The use of thin slices in a common practice in psychology [4] and social computing [29]. The comparison with predictive validity across slices is a research issue for future work.

The agreement between the raters was assessed using Intraclass Correlation Coefficient (ICC), a standard measure of inter-rater reliability widely used in psychology and social computing [32]. As all the raters annotated each video and because we used a sample rather than a population of raters, we used a two-way mixed, consistency, average-measures $\operatorname{ICC}(2, k)$. The agreement between raters for all the social variables was greater than 0.5 indicating moderate reliability. Table 1 summarizes the annotated social variables and presents their respective descriptive statistics.

As a first step, we analyzed the pairwise correlations (using Pearson's correlation) between the social variables. These are presented in Table 2. All variables annotated were observed to be significantly correlated with each other with correlation coefficients above 0.6 for all cases. Correlations between some variables like competent and hardworking $(r=0.91)$, sociable and enthusiastic $(r=$ $0.91)$, enthusiastic and positive $(r=0.96)$ were very high, indicating that they are essentially the same.

\section{NONVERBAL FEATURES}

Nonverbal cues were automatically extracted from the audio and visual modalities for both the applicant and interviewer towards the aim of obtaining behavioral representation of their interaction during job interviews. The choice of nonverbal cues extracted was based on their relevance in existing literature in the field of social 
Table 2: Correlation matrix for perceived social variables in job interviews $(\mathrm{N}=169)$. In all cases, correlation is significant (p<0.01).

\begin{tabular}{|c|c|c|c|c|c|c|c|c|c|}
\hline & 2 & 3 & 4 & 5 & 6 & 7 & 8 & 9 & 10 \\
\hline 1. ovImpression & 0.87 & 0.85 & 0.83 & 0.79 & 0.85 & 0.87 & 0.83 & 0.81 & 0.88 \\
\hline 2. motivated & & 0.85 & 0.84 & 0.76 & 0.83 & 0.84 & 0.79 & 0.65 & 0.74 \\
\hline 3. competent & & & 0.92 & 0.62 & 0.71 & 0.73 & 0.67 & 0.68 & 0.74 \\
\hline 4. hardworking & & & & 0.60 & 0.69 & 0.72 & 0.66 & 0.64 & 0.69 \\
\hline 5. sociable & & & & & 0.91 & 0.89 & 0.83 & 0.64 & 0.76 \\
\hline 6. enthusiastic & & & & & & 0.96 & 0.89 & 0.69 & 0.81 \\
\hline 7. positive & & & & & & & 0.90 & 0.73 & 0.81 \\
\hline 8. communicative & & & & & & & & 0.75 & 0.85 \\
\hline 9. concise & & & & & & & & & 0.88 \\
\hline 10. persuasive & & & & & & & & & \\
\hline
\end{tabular}

psychology [10, 19] and social computing [24, 25] and were extracted for the full interview. Analysis of other behavioral cues (e.g. gaze, facial expressions, verbal content) could also be extracted to get a comprehensive representation of the interaction and will be undertaken in future work.

\subsection{Audio Features}

Speaking Activity: cues such as pauses and speaking time are known to impact hireability impression ratings [23]. We extracted the following activity cues based on the speaker segmentation provided by the Microcone and utilized various statistics like count, mean, standard deviation, and maximum as features.

- Speaking time was obtained by summing all the individual speaking segments and normalized by interview duration.

- Speaking turn was defined as number of speech segments greater than two seconds.

- Pauses were defines as gaps in speech of less than two seconds in duration.

- Short utterances are speaking segments of duration less than two seconds.

Prosody Features: relate to the tonal variations of speech. This includes pitch (voice fundamental frequency), speaking rate (speed at which words are spoken), spectral entropy (measure of irregularity or complexity), energy (voice loudness), and time derivative of energy (voice loudness modulation). Speech feature were extracted using the code made available by MIT Media Lab [2]. We extracted the following statistics: mean, standard deviation, minimum, maximum, entropy, median, and quartiles.

\subsection{Visual Features}

Head Nods are vertical up-and down movements of the head, rhythmically raised and lowered. We used a 3D face centered method [7] in which a 3D head tracker calculates angular velocities using relative rotation at each instant with respect to the head pose at some instance before. Number of nods, mean, median, standard deviation, minimum, and maximum of duration were computed.

Overall Visual Motion We utilize a modified version of motion energy images, called Weighted Motion Energy Images (WMEI) [6] to capture the total amount of visual movement displayed by the applicant and interviewer during the job interview. We then compute various statistics: mean, median, standard deviation, minimum, maximum, entropy, quartiles, center of gravity, and utilize them as features.

\subsection{Multimodal Features}

Nodding while speaking was defined as events when a protagonist was nodding while he was speaking. Count of nodding while speaking, mean, median, standard deviation, minimum, and maximum of duration were computed.

\section{EXPERIMENTS AND RESULTS}

We divide our experiments into two sections, Section 5.1 describes correlation analysis of nonverbal behavior and social variables. In Section 5.2, we evaluate a computational framework for the automatic inference of first impressions from employment interviews. In Section 5.3, we present results comparing ratings and behavior between the first and second interviews.

\subsection{Correlation Analysis}

The results of correlation analysis for the social variables are presented in Table 3. Due to space constraints, only a few nonverbal cues are presented here. The complete correlation matrix can be found in the supplementary material.

Applicant cues: A number of applicant features were found to be significantly correlated to impressions of all social variables of interest. Specifically, participants who spoke often, longer, louder, with greater modulation of loudness and pitch obtained higher score in overall impression, professional, social and communicative skills This results are in accordance with existing literature [10, 12, 24].

Similarly, applicants who spoke animatedly with more gestures and motion were more favorably viewed than participants who spoke with less gestures. This corroborates the results in [24], which showed that applicants displaying more visual head motion (WMEI) received better hireability scores.

Interviewer cues: Interviewer's pitch (std), spectral entropy (min, lower quartile) and time derivative of energy (minimum) were observed to be negatively associated with all social variables suggesting that the interviewer had a more monotonous tone of voice in presence of job applicants who were rated higher. These results is in line with those reported in [10, 24].

Interviewer's overall visual motion (mean) was positively associated with overall impression and all social variables of interest. This suggests that interviewers gestured more in the presence of an applicant who scored higher than applicants with lower scores, thus again validating the findings in [24].

\subsection{Inference of First Impressions}

We formulate the prediction of social variables from extracted behavioral cues as a regression task. In the first step, we preprocessed the annotated data using log transformation due to skewness of data (skewness $>1$ ). Behavioral features too were normalized using the $\mathrm{z}$-score, such that they had zero mean and unity variance.

Dimensionality reduction techniques like Principle Component Analysis (PCA) and low $p$ value (pVal) were then evaluated. The $\mathrm{pVal}$ method assumes that relevant information is contained in the features significantly correlated with the social variables. Thus, we selected features with $p<0.05$. As the performance of $\mathrm{pVal}$ techniques was better then PCA, we report only the results of $\mathrm{pVal}$ technique. To test the impact of the dimensionality reduction step, we also tested the case of taking all features as predictors for the regression step. Several regression techniques (Ridge, random forest (RF), ordinary least squares (OLS)) were evaluated. For these tasks, we used leave-one-interview-out cross validation and 10-fold inner cross validation. As we used the leave-one-interview-out cross validation, it is possible that one participant can be in both the training and test sets although in different interviews.

For evaluation measure, we utilized the coefficient of determination $R^{2}$, which accounts for the amount of total variance explained by the model under analysis. This metric is often used in both psychology and social computing to evaluate regression tasks.

The results of the regression task are summarized in Table 4 . Results from utilizing all the data indicate that all social variables 
Table 3: Selected Pearson's correlation coefficient for various social variables. ${ }^{*} p<0.01, \dagger p<0.05$

\begin{tabular}{|c|c|c|c|c|c|c|c|c|c|c|}
\hline \multirow[t]{2}{*}{ Nonverbal Cues } & \multicolumn{3}{|c|}{ Professional Skills } & \multicolumn{3}{|c|}{ Social Skills } & \multicolumn{3}{|c|}{ Communication Skills } & \multirow{2}{*}{$\begin{array}{r}\text { Overall } \\
\text { ovImpress }\end{array}$} \\
\hline & motiv & compe & hardw & socia & enthu & posit & commu & conci & persu & \\
\hline $\begin{array}{l}\text { Applicant Speaking Activity } \\
\text { Avg Turn duration } \\
\text { Num Silent Events }\end{array}$ & $\begin{array}{r}0.38^{*} \\
-0.39^{*}\end{array}$ & $\begin{array}{r}0.27^{*} \\
-0.27^{*}\end{array}$ & $\begin{array}{r}0.27^{*} \\
-0.26^{*}\end{array}$ & $\begin{array}{r}0.39^{*} \\
-0.43^{*}\end{array}$ & $\begin{array}{r}0.41^{*} \\
-0.48^{*}\end{array}$ & $\begin{array}{r}0.39^{*} \\
-0.47^{*}\end{array}$ & $\begin{array}{r}0.38^{*} \\
-0.41^{*}\end{array}$ & $\begin{array}{r}0.20 \dagger \\
-0.32^{*}\end{array}$ & $\begin{array}{r}0.32^{*} \\
-035^{*}\end{array}$ & $\begin{array}{r}0.36^{*} \\
-0.42^{*}\end{array}$ \\
\hline $\begin{array}{l}\text { Applicant Pitch } \\
\text { Std Deviation } \\
\text { Lower quartile }\end{array}$ & $\begin{array}{r}-0.19 \dagger \\
0.21^{*}\end{array}$ & $\begin{array}{r}-0.15 \\
0.22^{*}\end{array}$ & $\begin{array}{r}-0.22^{*} \\
0.28^{*}\end{array}$ & $-0.21 \dagger$ & $\begin{array}{r}-0.28^{*} \\
0.26^{*}\end{array}$ & $\begin{array}{r}-0.29^{*} \\
0.27^{*}\end{array}$ & $\begin{array}{r}-0.22^{*} \\
0.19 \dagger\end{array}$ & $\begin{array}{r}-0.18 \dagger \\
0.17 \dagger\end{array}$ & -0.16 & $\begin{array}{r}-0.24^{*} \\
0.25^{*}\end{array}$ \\
\hline $\begin{array}{l}\text { Applicant Spectral Entropy } \\
\text { Average } \\
\text { Std Deviation }\end{array}$ & $\begin{array}{r}-0.14 \\
0.26^{*}\end{array}$ & $\begin{array}{r}-0.16 \dagger \\
0.29^{*}\end{array}$ & $\begin{array}{r}-0.20 \dagger \\
0.34^{*}\end{array}$ & $0.18 \dagger$ & $\begin{array}{r}-0.16 \dagger \\
0.30^{*}\end{array}$ & $\begin{array}{r}-0.18 \dagger \\
0.31^{*}\end{array}$ & $0.22^{*}$ & $\begin{array}{r}-0.23^{*} \\
0.21^{*}\end{array}$ & $0.17 \dagger$ & $\begin{array}{r}-0.23^{*} \\
0.28^{*}\end{array}$ \\
\hline $\begin{array}{l}\text { Applicant Energy } \\
\text { Average } \\
\text { Std Deviation } \\
\text { Lower quartile } \\
\text { Maximum }\end{array}$ & $\begin{array}{l}0.37^{*} \\
0.39^{*} \\
0.34^{*} \\
0.41^{*}\end{array}$ & $\begin{array}{l}0.24^{*} \\
0.27^{*} \\
0.27^{*} \\
0.30^{*}\end{array}$ & $\begin{array}{l}0.24^{*} \\
0.27^{*} \\
0.23^{*} \\
0.31^{*}\end{array}$ & $\begin{array}{l}0.33^{*} \\
0.37^{*} \\
0.32^{*} \\
0.40^{*}\end{array}$ & $\begin{array}{l}0.36^{*} \\
0.40^{*} \\
0.33^{*} \\
0.44^{*}\end{array}$ & $\begin{array}{l}0.36^{*} \\
0.40^{*} \\
0.31^{*} \\
0.43^{*}\end{array}$ & $\begin{array}{l}0.30^{*} \\
0.35^{*} \\
0.30^{*} \\
0.40^{*}\end{array}$ & $\begin{array}{l}0.19 \dagger \\
0.22^{*} \\
0.23^{*} \\
0.22^{*}\end{array}$ & $\begin{array}{l}0.28^{*} \\
0.31^{*} \\
0.33^{*} \\
0.33^{*}\end{array}$ & $\begin{array}{l}0.31^{*} \\
0.34^{*} \\
0.30^{*} \\
0.35^{*}\end{array}$ \\
\hline $\begin{array}{l}\text { Applicant Change in Energy } \\
\text { Maximum } \\
\text { Minimum }\end{array}$ & $\begin{array}{r}0.41^{*} \\
-0.40^{*}\end{array}$ & $\begin{array}{r}0.31^{*} \\
-0.27^{*}\end{array}$ & $\begin{array}{r}0.32^{*} \\
-0.30^{*}\end{array}$ & $\begin{array}{r}0.44^{*} \\
-0.42^{*}\end{array}$ & $\begin{array}{r}0.46^{*} \\
-0.44^{*}\end{array}$ & $\begin{array}{r}0.44^{*} \\
-0.43^{*}\end{array}$ & $\begin{array}{r}0.40^{*} \\
-0.40^{*}\end{array}$ & $\begin{array}{r}0.22^{*} \\
-0.20 \dagger\end{array}$ & $\begin{array}{r}0.33^{*} \\
-0.31^{*}\end{array}$ & $\begin{array}{r}0.35^{*} \\
-0.35^{*}\end{array}$ \\
\hline $\begin{array}{l}\text { Applicant WMEI } \\
\text { Maximum }\end{array}$ & $0.26^{*}$ & $0.26^{*}$ & $0.24^{*}$ & 0.15 & $0.17 \dagger$ & 0.15 & $0.18 \dagger$ & & & 0.16 \\
\hline $\begin{array}{l}\text { Interviewer Pitch } \\
\text { Average } \\
\text { Std Deviation }\end{array}$ & -0.15 & & & $\begin{array}{r}0.14 \\
-0.19 \dagger\end{array}$ & $\begin{array}{r}0.15 \\
-0.19 \dagger\end{array}$ & $\begin{array}{r}0.15 \\
-0.21 \dagger\end{array}$ & -0.14 & $-0.19 \dagger$ & $-0.19 \dagger$ & $\begin{array}{r}0.15 \\
-0.22^{*}\end{array}$ \\
\hline $\begin{array}{l}\text { Interviewer Spectral Entropy } \\
\text { Std Deviation } \\
\text { Minimum }\end{array}$ & $\begin{array}{r}0.17 \dagger \\
-0.21^{*}\end{array}$ & $\begin{array}{r}0.15 \\
-0.16\end{array}$ & & $\begin{array}{r}0.23^{*} \\
-0.23^{*}\end{array}$ & $\begin{array}{r}0.24^{*} \\
-0.25^{*}\end{array}$ & $\begin{array}{r}0.24^{*} \\
-0.26^{*}\end{array}$ & $\begin{array}{c}0.17 \dagger \\
-0.14\end{array}$ & $\begin{array}{r}0.19 \dagger \\
-0.21^{*}\end{array}$ & $\begin{array}{r}0.20 \dagger \\
-0.18 \dagger\end{array}$ & $\begin{array}{r}0.24^{*} \\
-0.26^{*}\end{array}$ \\
\hline $\begin{array}{l}\text { Interviewer Energy } \\
\text { Std Deviation } \\
\text { Maximum }\end{array}$ & $\begin{array}{l}0.28^{*} \\
0.28^{*}\end{array}$ & $\begin{array}{l}0.19 \dagger \\
0.21^{*}\end{array}$ & $\begin{array}{l}0.19 \dagger \\
0.23^{*}\end{array}$ & $\begin{array}{l}0.24^{*} \\
0.25^{*}\end{array}$ & $\begin{array}{l}0.25^{*} \\
0.29^{*}\end{array}$ & $\begin{array}{l}0.25^{*} \\
0.27^{*}\end{array}$ & $\begin{array}{l}0.25^{*} \\
0.26^{*}\end{array}$ & & $\begin{array}{r}0.16 \\
0.19 \dagger\end{array}$ & $\begin{array}{l}0.19 \dagger \\
0.21^{*}\end{array}$ \\
\hline $\begin{array}{l}\text { Interviewer Change in Energy } \\
\text { Maximum } \\
\text { Minimum }\end{array}$ & $\begin{array}{r}0.28^{*} \\
-0.27^{*}\end{array}$ & $\begin{array}{r}0.24^{*} \\
-0.19 \dagger\end{array}$ & $\begin{array}{r}0.24^{*} \\
-0.21^{*}\end{array}$ & $\begin{array}{r}0.25^{*} \\
-0.27^{*}\end{array}$ & $\begin{array}{r}0.29^{*} \\
-0.29^{*}\end{array}$ & $\begin{array}{r}0.28^{*} \\
-0.27^{*}\end{array}$ & $\begin{array}{r}0.26^{*} \\
-0.25^{*}\end{array}$ & 0.14 & $\begin{array}{r}0.20 \dagger \\
-0.19 \dagger\end{array}$ & $\begin{array}{r}0.22^{*} \\
-0.21^{*}\end{array}$ \\
\hline $\begin{array}{l}\text { Interviewer WMEI } \\
\text { Average }\end{array}$ & $0.23^{*}$ & $0.21^{*}$ & $0.19 \dagger$ & & 0.15 & 0.15 & 0.15 & 0.14 & & 0.16 \\
\hline
\end{tabular}

annotated were predictable to some degree from nonverbal behavior. Random forest with $\mathrm{pVal}$ dimensionality reduction (pVal-RF) was found to perform best with this set of features. Overall impression and enthusiastic had the highest $R^{2}, 0.34$ and 0.32 respectively. This implies nonverbal behavior is predictive of overall first impression as shown in existing literature [20] and corroborates the results found in [24]. We observe certain variables like hardworking $\left(R^{2}=0.15\right)$ and competence $\left(R^{2}=0.18\right)$ to be harder to predict.

Comparing to recent work, in [24], the authors reported $R^{2}$ of 0.36 for hireability, a measure we have not used. They also reported $R^{2}$ of 0.10 for persuasive and no predictability for communicative. In another work, [23] reported results on a different set of social variables using correlation coefficient $r$ as their evaluation metric. We compare our results to this work by converting $r$ to $R^{2}$ (our evaluation metric, coefficient of determination $R^{2}$ is obtained by computing the square of correlation coefficient $r$ ). They reported a prediction accuracy of $r=0.70$ for overall performance, which indicates a $R^{2}$ of 0.49 . We compare results of socials constructs which are similar in meaning to the ones we have utilized: excited $\left(R^{2}=0.65\right)$ vs enthusiastic $\left(R^{2}=0.34\right)$, friendly $\left(R^{2}=0.63\right)$ vs social $\left(R^{2}=0.19\right)$, focused $\left(R^{2}=0.31\right)$ vs motivated $\left(R^{2}=0.29\right)$. There is no direct way of assessing where the performance difference come from, as the data set used Naim et al. is not publicly available to our knowledge.

We observe that language has an effect on the predictive power of impressions scores. A larger variance for overall impression $\left(R^{2}=0.32\right)$ could be explained for participants using French as the language of interview, while for participants using English only $14 \%$ of variance could be explained. We hypothesize that this could be due to the fact that raters were not native English speakers. The same hypothesis could explain why concise had higher $R^{2}$ for English than French. However, the small size of the English dataset prevents drawing firm conclusions on the effect of language and will be investigated.

Although no significant difference could be observed between males and females in terms of the values of annotations, we observe that the interviews including a male participant were predicted with higher accuracy $\left(R^{2} \in[0.13,0.46]\right)$ than the ones featuring females $\left(R^{2} \leq 0.12\right)$. In order to understand these differences, we analyzed the correlations between nonverbal cues and the annotated variables for data subsets separated based on gender. Table 5 displays a summary of the largest differences in correlation values for the variable of overall impression; due to space constraints, we did not include the correlations for other variables, but similar trends were found. We observe that the improvement in prediction accuracy can be explained by the overall higher correlations observed for male interviews. Furthermore, striking gender differences can be observed in terms of correlation values, such as speaking time, statistics of turn duration, speech energy, and silence; these behaviors are part of what psychologists refer to as powerful speech. On the one hand, if men use these cues they are perceived as powerful, which is in line with gender stereotypes for men, as well as with persuasiveness [21]. On the other hand, stereotpyically women are less expected to show powerful speech, which might explain the lower correlations found for women.

\subsection{Changes Across Sessions}

To determine if there was a difference in annotated ratings across sessions, we selected only those participants who had completed 
Table 4: Regression results for each language and gender using regression methods; $p$ Val with random forest (pVal-RF) and pVal with Ridge (pVal-Ridge) ${ }^{*} p<0.01, \dagger p<0.05$.

\begin{tabular}{|c|c|c|c|c|c|c|c|c|c|c|}
\hline Nonverbal Cues & \multicolumn{2}{|c|}{ All $(N=169)$} & \multicolumn{2}{|c|}{ English $(N=39)$} & \multicolumn{2}{|c|}{ French $(N=130)$} & \multicolumn{2}{|c|}{ Female $(N=96)$} & \multicolumn{2}{|c|}{ Male $(N=73)$} \\
\hline & Method & $R^{2}$ & Method & $R^{2}$ & Method & $R^{2}$ & Method & $R^{2}$ & Method & $R^{2}$ \\
\hline Overall Impression & pVal-RF & $0.32^{*}$ & pVal-Ridge & $0.14 \dagger$ & pVal-RF & $0.32^{*}$ & pVal-Ridge & 0.06 & pVal-RF & $0.44^{*}$ \\
\hline Communication & pVal-RF & $0.25^{*}$ & pVal-Ridge & $0.07 \dagger$ & pVal-Ridge & $0.16 \dagger$ & pVal-Ridge & -0.07 & pVal-Ridge & $0.45^{*}$ \\
\hline Persuasive & pVal-RF & $0.20 \dagger$ & pVal-Ridge & $0.09 \dagger$ & pVal-RF & $0.20 \dagger$ & pVal-RF & 0.05 & pVal-RF & $0.28 \dagger$ \\
\hline Concise & pVal-RF & $0.14 \dagger$ & pVal-Ridge & $0.38 \dagger$ & pVal-Ridge & -0.06 & pVal-Ridge & -0.13 & Pval-RF & $0.13 \dagger$ \\
\hline Enthusiastic & pVal-RF & $0.34^{*}$ & pVal-RF & $0.12 \dagger$ & pVal-RF & $0.31^{*}$ & pVal-Ridge & 0.07 & pVal-Ridge & $0.46^{*}$ \\
\hline Positive & pVal-RF & $0.30^{*}$ & pVal-RF & $0.06 \dagger$ & pVal-RF & $0.27 \dagger$ & pVal-Ridge & 0.12 & pVal-Ridge & $0.44^{*}$ \\
\hline Social & pVal-RF & $0.19^{*}$ & pVal-RF & $0.14 \dagger$ & pVal-RF & 0.15 & pVal-Ridge & 0.06 & pVal-Ridge & $0.44^{*}$ \\
\hline Competence & pVal-RF & 0.18 & pVal-Ridge & $0.15 \dagger$ & pVal-RF & $0.22 \dagger$ & pVal-Ridge & -0.10 & pVal-Ridge & $0.38^{*}$ \\
\hline Hardworking & pVal-RF & 0.15 & pVal-Ridge & -0.24 & pVal-RF & 0.12 & pVal-Ridge & -0.18 & pVal-RF & $0.44^{*}$ \\
\hline Motivated & pVal-RF & $0.29^{*}$ & pVal-RF & $0.26 \dagger$ & pVal-RF & $0.27 \dagger$ & pVal-Ridge & 0.04 & pVal-RF & $0.44^{*}$ \\
\hline
\end{tabular}

Table 5: Correlation coefficients between selected nonverbal cues and overall impression for sub-sets separated based on gender. ${ }^{*} p<0.01 ; \dagger p<0.05$

\begin{tabular}{lcc}
\hline & \multicolumn{2}{c}{ Overall impression } \\
NVB cues & Female $(N=96)$ & Male $(N=73)$ \\
\hline App. \# of turns & $-0.36^{*}$ & $-0.42^{*}$ \\
App. speaking time & - & $0.23 \dagger$ \\
App. speaking ratio & - & $0.35^{*}$ \\
App. turn duration stats & $0.25 \dagger$ & {$[0.37,0.48]^{*}$} \\
App. speech energy stats & {$[0.17,0.26] \dagger$} & {$[0.42,0.50]^{*}$} \\
Silence stats & $-0.23^{*}$ & {$[-0.47,-0.67]^{*}$} \\
\hline
\end{tabular}

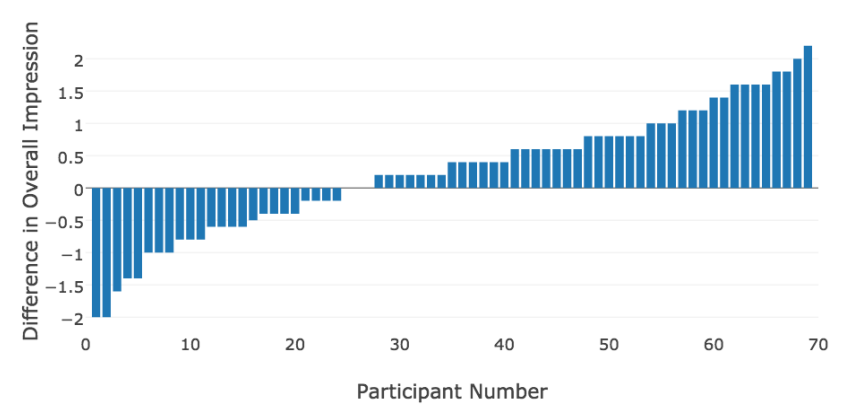

Figure 4: Difference in Overall Impression score between two lab sessions for all participants.

both lab session 1 and $2(N=69)$ from the full dataset. We then conducted a paired t-test on this split dataset, which rejected the null hypothesis for overall impression $(\mathrm{p}<0.05)$. Improvement of scores for other social variables were also significant $(p<0.05)$.

Similarly, we conducted a similar experiment to determine if there was a difference in nonverbal behavior across sessions. We observed that there was a significant $(p<0.01)$ change in applicant maximum turn duration and speaking time. Correlation between the change in overall impression scores and change in speaking activity was statistically significant. Table 6 summarizes the descriptive statistics of social variables and nonverbal behaviors which changed significantly between lab session 1 and 2 . The difference in overall impression between the two lab sessions for each participant can be visualized in Figure 4. We observe that while the majority of the participants had an improvement in their ratings, 34\% of participants had a decrease in scores between in the lab session 2 and lab session 1 . There was no changes in scores for 4 participants. Although we observe that students overall improved their interview performance at the second laboratory session, no conclusion can be drawn about the factors that were favorable to the student's behavioral improvement. For instance, the source of the
Table 6: Descriptive stats of social variables and nonverbal cues. Mean values for speaking cues are after z-score normalization.

\begin{tabular}{lcccc}
\hline & \multicolumn{2}{c}{ Mean Value } & & \\
Nonverbal cues & Session 1 & Session 2 & Delta & pValue \\
\hline Overall Impression & 5.2 & 5.5 & 0.3 & 0.03 \\
Communicative & 5.5 & 5.7 & 0.2 & 0.03 \\
Sociable & 5.2 & 5.4 & 0.2 & 0.02 \\
Persuasive & 5.2 & 5.5 & 0.3 & 0.01 \\
\hline Applicant Speaking Time & 0.60 & 0.66 & 0.6 & 0.008 \\
Applicant Turn duration & 0.11 & 0.13 & 0.02 & 0.005 \\
\hline
\end{tabular}

improvement in interview performance could be due to the feedback they were given, but also to the fact that they participated to the role-play a second time, in which their level of confidence was higher. In future work, we plan to understand the factors that encourage behavioral improvement: What is the best way to display feedback? What behaviors should feedback focus on? Who are the students that benefit from feedback, e.g. in terms of personality, gender, age? We believe that this work constitutes a first step towards addressing these research questions.

\section{DISCUSSION AND CONCLUSION}

In this work, we described a number of challenges associated with implementing a behavioral training procedure for hospitality students in order to improve the first impressions that others form about them. The framework consisted of two scenarios that were relevant for their future careers, namely job interviews and reception desk situations. We collected a new corpus of 169 simulated job interviews and reception desk interactions (338 interactions). This dataset was recorded with multiple modalities.

Nonverbal cues were automatically extracted and their relationship with various perceived social variables analyzed. Our results are comparable with those reported in [24] and [23].

One of the insights from our analysis is that language has an effect on the predictive power of impressions scores. Using data from only French language interviews showed higher prediction accuracy $\left(R^{2}=0.32\right)$ than interviews which were conducted in only English $\left(R^{2}=0.14\right)$ for overall impression, while accuracy for other social variables (except "concise") was comparable. Understanding these issues in more depth requires future work.

Another insight was the role of gender in prediction accuracy. Interviews with male participant were predicted with higher accuracy $\left(R^{2} \in[0.13,0.46]\right)$ than the ones featuring females $\left(R^{2} \leq 0.12\right)$. As the difference in $N$ is relatively small (57\% female to $43 \%$ male) and both data subsets have comparable size (or larger) compared to other existing datasets, we believe this is an interesting result. This result is supported by findings in psychology [21] and is in line with gender stereotypes for men, and with persuasiveness. 
The presented work is a first step towards the implementation of a living lab in a hospitality management school. The use of the part of the corpus not analyzed here will allow us to analyze a second common situation in hospitality and to provide improved feedback to the student population.

\section{ACKNOWLEDGMENTS}

This work was funded by the UBIMPRESSED project of the Sinergia interdisciplinary program of the Swiss National Science Foundation (SNSF). We thank the students of Vatel Switzerland for their participation, and Vatel's academic deans (Susanne Welle, Alexandra Broillet, Daniel Fuchs, and Marc Forestier) for their support. We also thank Tanzeem Choudhury (Cornell University) for discussions, and Dayra Sanchez, Ailbhe Finnerty, and the research assistants for their help with data collection.

\section{REFERENCES}

[1] http://www.dev-audio.com/products/microcone/.

[2] http://groupmedia.media.mit.edu/data.php.

[3] N. Ambady, M. Hallahan, and R. Rosenthal. On judging and being judged accurately in zero-acquaintance situations. $J$. Personality and Social Psychology, 69(3), 1995.

[4] N. Ambady and R. Rosenthal. Thin slices of expressive behavior as predictors of interpersonal consequences: A meta-analysis. Psychological bulletin, 111(2), 1992.

[5] L. M. Batrinca, N. Mana, B. Lepri, F. Pianesi, and N. Sebe. Please, tell me about yourself: automatic personality assessment using short self-presentations. In Proc. ACM ICMI, 2011.

[6] J.-I. Biel and D. Gatica-Perez. The youtube lens: Crowdsourced personality impressions and audiovisual analysis of vlogs. IEEE Trans. on Multimedia, 15(1), 2013.

[7] Y. Chen, Y. Yu, and J.-M. Odobez. Head nod detection from a full 3d model. In Proc. IEEE ICCV Workshops, 2015.

[8] J. R. Curhan and A. Pentland. Thin slices of negotiation: predicting outcomes from conversational dynamics within the first 5 minutes. J. Applied Psychology, 92(3), 2007.

[9] I. Damian, C. S. S. Tan, T. Baur, J. Schöning, K. Luyten, and E. André. Augmenting social interactions: Realtime behavioural feedback using social signal processing techniques. In Proc. ACM CHI, 2015.

[10] T. DeGroot and J. Gooty. Can nonverbal cues be used to make meaningful personality attributions in employment interviews? J. Business and Psychology, 24(2), 2009.

[11] T. DeGroot and S. J. Motowildo. Why visual and vocal interview cues can affect interviewers' judgments and predict job performance. J. Applied Psychology, 84(6), 1999.

[12] C. M. End and K. Saunders. Short communication: Powerless and jobless? comparing the effects of powerless speech and speech disorders on an applicant's employability. Frontiers, 2(1), 2013.

[13] R. J. Forbes and P. R. Jackson. Non-verbal behaviour and the outcome of selection interviews. J. Occupational Psychology, 53(1), 1980.

[14] D. Gatica-Perez. Automatic nonverbal analysis of social interaction in small groups: A review. Image and Vision Computing, 27(12), 2009.

[15] D. Gatica-Perez. Signal processing in the workplace. IEEE Signal Process. Mag., 32(1), 2015.
[16] J. G. Hollandsworth, R. Kazelskis, J. Stevens, and M. E. Dressel. Relative contributions of verbal, articulative, and nonverbal communication to employment decisions in the job interview setting. J. Personnel Psychology, 32(2), 1979.

[17] M. E. Hoque, M. Courgeon, J.-C. Martin, B. Mutlu, and R. W. Picard. Mach: My automated conversation coach. In Proc. ACM UBICOMP, 2013.

[18] A. I. Huffcutt, J. M. Conway, P. L. Roth, and N. J. Stone. Identification and meta-analytic assessment of psychological constructs measured in employment interviews. J. Applied Psychology, 86(5), 2001.

[19] A. S. Imada and M. D. Hakel. Influence of nonverbal communication and rater proximity on impressions and decisions in simulated employment interviews. J. Applied Psychology, 62(3), 1977.

[20] M. Knapp, J. Hall, and T. Horgan. Nonverbal communication in human interaction. Cengage Learning, 2013.

[21] C. Leaper and R. D. Robnett. Women Are More Likely Than Men to Use Tentative Language, Aren't They? A Meta-Analysis Testing for Gender Differences and Moderators. Psychology of Women Quarterly, 35(1), 2011.

[22] T. V. McGovern and H. E. Tinsley. Interviewer evaluations of interviewee nonverbal behavior. J. Vocational Behavior, 13(2), 1978.

[23] I. Naim, M. I. Tanveer, D. Gildea, and M. E. Hoque. Automated prediction and analysis of job interview performance: The role of what you say and how you say it. Proc. IEEE FG, 2015.

[24] L. S. Nguyen, D. Frauendorfer, M. S. Mast, and D. Gatica-Perez. Hire me: Computational inference of hirability in employment interviews based on nonverbal behavior. IEEE Trans. on Multimedia, 16(4), 2014.

[25] L. S. Nguyen and D. Gatica-Perez. I would hire you in a minute: Thin slices of nonverbal behavior in job interviews. In Proc. ACM ICMI, 2015.

[26] L. S. Nguyen, A. Marcos-Ramiro, M. Marrón Romera, and D. Gatica-Perez. Multimodal analysis of body communication cues in employment interviews. In Proc. ACM ICMI, 2013.

[27] E. Ofek, S. T. Iqbal, and K. Strauss. Reducing disruption from subtle information delivery during a conversation: mode and bandwidth investigation. In Proc. ACM CHI, 2013.

[28] A. Pentland and T. Heibeck. Honest signals: how they shape our world. MIT press, 2010.

[29] F. Pianesi, N. Mana, A. Cappelletti, B. Lepri, and M. Zancanaro. Multimodal recognition of personality traits in social interactions. In Proc. ACM ICMI, 2008.

[30] S. Rothmann and E. P. Coetzer. The big five personality dimensions and job performance. J. Industrial Psychology, 29(1), 2003.

[31] B. R. Schlenker. Impression management: The self-concept, social identity, and interpersonal relations. Brooks/Cole Publishing Company Monterey, CA, 1980.

[32] P. E. Shrout and J. L. Fleiss. Intraclass correlations: uses in assessing rater reliability. Psychological bulletin, 86(2), 1979.

[33] M. I. Tanveer, E. Lin, and M. E. Hoque. Rhema: A real-time in-situ intelligent interface to help people with public speaking. In Proc. of Int Conf. on Intelligent User Interfaces. ACM, 2015. 